\title{
Theoretical Study of the Electron Transport through Aromatic Molecular Wires with Different Aromatic Rings
}

\author{
Z. BAYAT ${ }^{a, *}$ AND E. TAGHIZADEH ${ }^{b}$ \\ ${ }^{a}$ Department of Chemistry, Quchan Branch, Islamic Azad University, Quchan, Iran \\ ${ }^{b}$ Department of Chemistry, Young Researchers Club, Quchan Branch, Islamic Azad University, Quchan, Iran \\ (Received January 20, 2017; revised version October 2, 2017; in final form October 22, 2017)

\begin{abstract}
Designing molecular systems for molecular electronics or for solar energy conversion that are capable of moving charge efficiently over long distances through molecular bridges requires a fundamental understanding of electron transport in donor-bridge-acceptor systems. In this paper theoretical investigation was performed on electron transport properties of 4-amino 4-nitro biphenyl (DBA-based molecule that was sandwiched between two gold surfaces). Dependence of the molecular electronic structure of the gold-molecule complex on the external electric field was studied, too. On the other hand, the electronic conduction was analyzed from the change in the shape of molecular orbital and the evolution of the highest occupied-lowest occupied molecular orbitals gap of the gold-molecule complex under the influence of the electric field. Values of potential barrier that were determined experimentally and the obtained results are reported in this paper.
\end{abstract}

DOI: 10.12693/APhysPolA.133.179

PACS/topics: Electron transport, aromatic, molecular wires, 4-amino 4-nitro biphenyl

\section{Introduction}

The experimental demonstration of the conceptually fundamental molecular electronic components like molecular wires, molecular rectifiers, and molecular switches $[1-5]$ can be considered as major step towards the "post-silicon technology". In all these molecular rectifiers it remains as one of the central attractions of molecular electronics research [6-11]. As discussed in paper, it is clear that donor (D)/acceptor (A) substituted allenic molecules is a useful alternate for the present days rectifiers. As in case of junction diode, the $p n$-junction part controls the electron flow; here in the donor-bridgeacceptor (DBA) type of molecular rectifier, the bridging unit between the $\mathrm{D}$ and $\mathrm{A}$ controls the flow of electron. In view of the potential rectifying ability of the $\mathrm{D} / \mathrm{A}$ substituted allenic molecule here in this paper, a detailed analysis on the effect of the allenic bridge placed in the electron transfer path between the donor and acceptor, has been carried out through frontier molecular orbital (FMO) analysis.

\section{Computational detail}

The detailed analysis of the structure-property correlation while considering various perturbing effects like $\mathrm{D} / \mathrm{A}$ effect, effect of hetero atom substitution in the allenic bridge, vinyl conjugation, etc., was carried out and all these results were compared with the reported DBA type of rectifiers.

References to the Bhanuprakash article donor and acceptor linkers have influence on bridges the same as this

\footnotetext{
*corresponding author; e-mail: z.bayat@ymail.com
}

paper. A particular challenge stems from the realization that the properties of molecular wires are strongly influenced by (I) characteristics of the molecule (II) nature of linkage and thus metal-molecule interaction. To achieve the transport property, theoretical analysis of the frontier molecular orbital will give a clear picture of the structure-property relationship. Molecule DB1ADB11A considered are shown in Fig. 1 and the corresponding optimized molecular geometric parameters around the ring junctions for molecules DB1A-DB11A are shown in Table I.

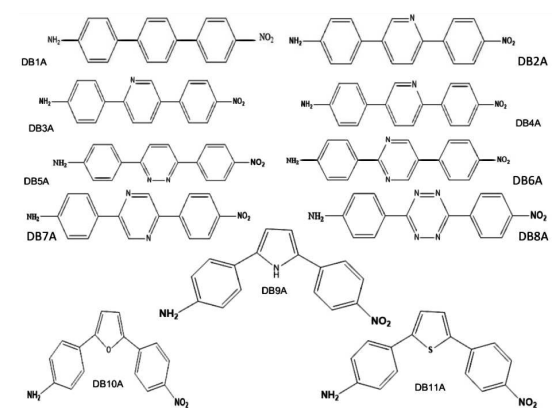

Fig. 1. DBA is composed of 3 rings and NH2, NO2 linkers (donor, acceptor) from both sides of molecules is attached to the benzene ring that has different bridges.

It is well recognized that the energy difference between highest occupied molecular orbital (HOMO) and the lowest unoccupied molecular orbital (LUMO) known as HOMO-LUMO gap (HLG) (Tables II and III) is a key parameter determining (Figs. 2 and 3) the conductance property [12-14]. Our approach is to analyze the role of aromatic $\pi$-bridges on electron transport property in a DBA system through frontier molecular orbital analysis. 
TABLE I

Optimized bond lengths $R_{i}[\AA]$ and twist angles $D_{i}\left[{ }^{\circ}\right]$ along the each ring junction for molecule DB1A-DB11

\begin{tabular}{c|c|c|c|c}
\hline \hline Mol. & $R_{1}$ & $D_{1}$ & $R_{2}$ & $D_{2}$ \\
\hline D B1 A & 1.48016 & -35.16999 & 1.48069 & -35.2582 \\
D B2 A & 1.47620 & -34.51412 & 1.48543 & -15.07687 \\
D B3 A & 1.47797 & 10.89558 & 1.47695 & 35.24311 \\
D B4 A & 1.47297 & -33.84643 & 1.48416 & -0.16714 \\
D B5 A & 1.46932 & -0.71830 & 1.47430 & -35.21194 \\
D B6 A & 1.47197 & -14.14660 & 1.48000 & -16.02357 \\
D B7 A & 1.47434 & -13.14653 & 1.48289 & 16.44992 \\
D B8 A & 1.46011 & -0.00146 & 1.47690 & 0.07601 \\
D B9 A & 1.46005 & -24.85776 & 1.45045 & 18.04467 \\
D B10 A & 1.45220 & -0.43128 & 1.44721 & 0.09291 \\
D B11 A & 1.46274 & -23.88989 & 1.45983 & -15.95370
\end{tabular}

TABLE II

HOMO, LUMO and HLG levels [eV] for free molecule.

\begin{tabular}{c|c|c|c}
\hline \hline Mol. & HOMO & LUMO & HLG \\
\hline H2N B1 NO2 & -5.46542774 & -2.344978349 & 3.120459391 \\
H2N B2 NO2 & -5.61262922 & -2.399221135 & 3.213408085 \\
H2N B3 NO2 & -5.486153578 & -2.449375269 & 3.036778309 \\
H2N B4 NO2 & -5.770723774 & -2.478268412 & 3.292455362 \\
H2N B5 NO2 & -5.586734423 & -2.562222071 & 3.024512352 \\
H2N B6 NO2 & -5.64942709 & -2.538235312 & 3.111191778 \\
H2N B7 NO2 & -5.673141273 & -2.5289677 & 3.144173573 \\
H2N B8 NO2 & -5.950897049 & -2.802262264 & 3.148534785 \\
H2N B9 NO2 & -5.105363766 & -2.235129892 & 2.870233874 \\
H2N B10 NO2 & -5.207852648 & -2.322081896 & 2.885770752 \\
H2N B11 NO2 & -5.34986517 & -2.395132483 & 2.954732687
\end{tabular}

Molecular orbitals of 11 complexes systems offer a unique context for the study of intra molecular charge transfer (charge delocalization) within molecules (Fig. 2). The property arises after one-electron of donor$p$ (bridge)-donor molecules, where a competition for the positive charge occurs between the donors through the $p$ -

TABLE III

Calculated distance between $\mathrm{Au}$ and the linker $\mathrm{L}: d_{\alpha}$ for triangle $\mathrm{Au}$ and $d_{\beta}$ for lozenge $\mathrm{Au}[\AA]$, binding energy $E_{b}$, HOMO, LUMO and HLG energies [eV] for 11 complexes.

\begin{tabular}{|c|c|c|c|c|c|c|}
\hline Comp. & $d_{\alpha}$ & $d_{\beta}$ & $E_{b}$ & $E_{H O M O}$ & $E_{L U M O}$ & $E_{H L G}$ \\
\hline $\mathrm{Au}$ N B1 NO2 Au & 27711 & 26649 & $|-1.488|$ & -5.406 & -3.648 & 1.758 \\
\hline $\mathrm{Au} N \mathrm{~B} 2 \mathrm{NO} 2 \mathrm{Au}$ & 37272 & 13580 & $|-1.814|$ & -5.638 & -3.799 & 1.839 \\
\hline $\mathrm{Au}$ N B3 NO2 Au & .36269 & 4.16934 & $-1.752 \mid$ & -5.526 & -3.934 & 1.592 \\
\hline $\mathrm{Au}$ N B4 & 36 & 4.0 & -1.837 & -5.628 & -3.785 & 1.843 \\
\hline Au N B5 & 36 & 4.10680 & $-1.880 \mid$ & -5.548 & -3.896 & 1.732 \\
\hline $\mathrm{Au}$ N B 6 NO2 Au & 1.373 & 4.05375 & $|-1.869|$ & -5.639 & -3.816 & 1.823 \\
\hline $\mathrm{Au}$ & 1.36125 & 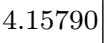 & -1.741 & -5.609 & -3.936 & 1.673 \\
\hline $\mathrm{Au}$ N B8 NO2 Au & 1.36880 & 3.98664 & -1.850 & -5.695 & -3.895 & 1.8 \\
\hline $\mathrm{Au}$ N B9 NO2 Au & $1.35^{\prime}$ & 3.98183 & $|-1.652|$ & -5.444 & -3.908 & 1.536 \\
\hline $\mathrm{B} 1 \mathrm{C}$ & 1.36 & .9129 & -1.733 & -5.521 & -3.852 & 1.669 \\
\hline $\mathrm{Au}$ N B11 NO2 Au & 2.95725 & 4.40735 & $-1.741 \mid$ & -5.648 & -4.141 & 1.507 \\
\hline
\end{tabular}

conjugated bridge. We can have two views (I, II). I: with full charge delocalization between the donors through the bridge, and II: where the charge resides on one of the donors and the bridge stops delocalization conversely.
$\mathrm{HOMO}$

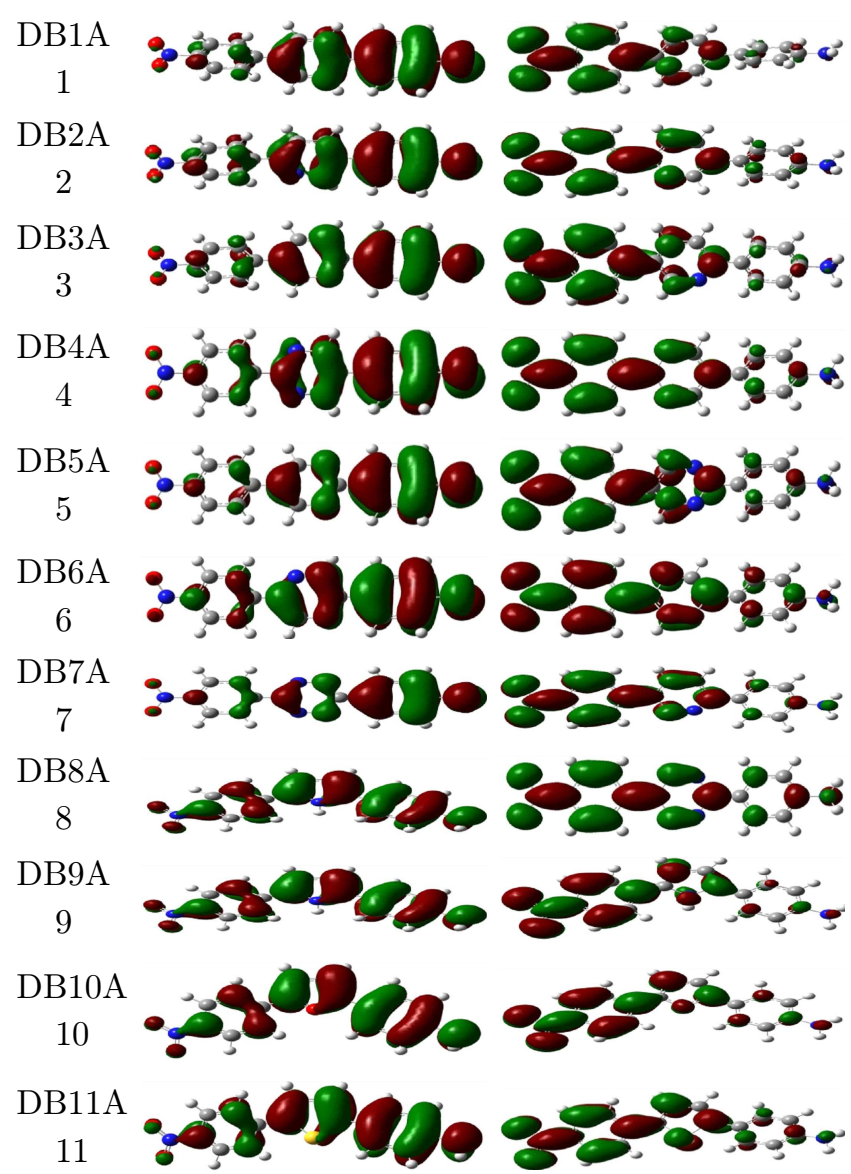

Fig. 2. The shape of HOMO and LUMO orbital fore the free molecule.

Representations of the molecular orbitals for the HOMO and LUMO electronic states for compounds are presented in Fig. 1, which show the typical delocalization of the charge of a conjugated molecule, using $p$-type orbitals along the complete structure. Therefore, depending on if the charge transport is through the HOMO or the LUMO orbital, the conductance of both molecules could be different. Based on the proximity of the HOMO energies of both molecules, as obtained from electrochemistry, with the Fermi level of gold, as a first approximation, the injection could be through the HOMO.

\section{Principle of electrical rectification}

The principle of the electrical rectification and electron transport characteristics of such types of molecules are well explained by Ellenbogen and Love in a simple manner by considering the FMOs which are responsible for the electron transport through the molecule [15]. As explained in the article by Ellenbogen and Love [15], in a proper applied bias voltage, electron tunneling occurs 
from the acceptor part of the molecule to the donor part, through the unoccupied manifold of the molecular orbitals. When such a molecule is connected through two electrodes (acceptor part connected to the cathode and donor part connected to the anode) and a proper forward bias (at least it should be sufficient enough to raise the Fermi energy of the electron in the occupied level of gold contact on the acceptor side to that of the energy of the LUMO localized on the acceptor part of the molecule) is applied, then the electron can flow from the gold contact to the LUMO, localized on the acceptor part. The injected electron from the gold contact to the acceptor part of the molecule can now tunnel through the central insulating bridge to the higher unoccupied molecular orbital localized in the donor half of the molecule and finally escapes into the gold contact lying in the donor part. In analogy to the forward bias condition, in a reverse applied bias, for electron transfer to occur from the donor part of the molecule to the acceptor part through the central insulating bridge, the reverse bias must be sufficient to raise the Fermi energy of the gold contact on the donor side of the molecule so that it is at least as high as the energy of the empty orbital localized on the donor part of the molecule. According to Ellenbogen and Love [15], during the process of applied bias in the forward and reverse bias conditions, energy level alignment occurs in the molecule which is responsible for a feasible electron transfer process in the forward bias condition compared to reverse biased condition (reverse biased process requires more energy than for the forward biased), and thus rectification occurs.

\section{Methodology}

Full geometry optimization of these structures is carried out within density functional theory (DFT), using the Gaussian 03 package. The B3LYP exchangecorrelation functional and a $6-31 \mathrm{G}^{*}$ basis set for nonmetal groups and LANL2DZ for the metal atoms (the gold atoms) are applied.

Each gold cluster consisted of three gold atoms that were placed as an equilateral triangle with sides of $2.88 \AA[16]$; the relative positions of the gold atoms were fixed in triangle and lozenge. The linker was positioned in the hollow site of the gold cluster and the distance between the two gold surfaces was fixed in space and all other geometric parameters were optimized. To obtain the ground state distance between the gold surface and the linker, we performed a series of calculations varying the distance between 1.90 and $2.39 \AA$ and the minima were found for each complex. In the final, the two gold surfaces were fixed in the space and the electric field $\left(E_{F}\right)$ of magnitude zero to $0.103 \mathrm{~V} / \AA$ aligned along the Au3linker-DBA-linker-Au3 inter atomic vector was applied to each complex that could reasonably represent the working condition of the molecular wire.

On the other hand, some researchers have studied the effects of high magnitude of electric field in experiment. For example the polycrystalline manganite samples were studied under high magnetic fields. The result is that at higher magnetic fields, the spins are aligned, the magnetization approaches saturation, and external magnetic field has weak effect on the transport properties: the magnetoresistance increase rate substantially reduces (usually, linearly) [17-19].

\section{Interaction with gold cluster}

The electrical performance of a molecule sandwiched between two metal electrodes is predominated by many factors, such as the nature of the molecule itself [20], the interface between the molecule and electrode [21], the electrode material [22], and the electrode shape [23]. The interaction between the molecule and the electrode also includes attaching two small gold clusters [24, 25].

Many researchers have used small golden clusters as electrodes in their research and have achieved good results. Many researchers have used golden clusters as electrodes in their research and have achieved good results. Briere et al., for example, have used small clusters of metals as electrods, including a gold triple cluster, and has gained promising results. Another scholar who studied small golden clusters was Prashant. Prashant et al., have studied the low-energy electronic structures and properties of small gold clusters [26, 27].

In this regard, it is of utmost importance to study and understand charge transport through a single molecular chain and to correlate it with its specific chemical structure and associated electronic properties in order to optimize its conductance $[28,29]$. The terminated atoms are always confined at the centers of the gold triangles, that is, the hollow site of the gold clusters, the orientation, and the structures of the complexes were obtained. We firstly performed a geometric optimization at distance between the two gold triangles and the terminated atom in each complex [30]. The optimized geometry of A-DB-Au showed where DBA remains a planar conformation and is perpendicular to the gold triangles in all eight complexes. We compared just the bond distance between terminal atom and $\mathrm{Au}$ because the geometries for other atoms of the phenyl rings are barely affected [31]. Table III presents the energetic and typical geometrical parameters for the energetically optimal distance between the terminated atom and the gold surfaces. Also we concentrate our attention on the relationship between the conductance change and the location of molecular orbital's (MOs), especially the HOMOs and the LUMOs. In this way these the shapes of HOMO and LUMO orbital's for complexes are shown in Fig. 3.

The binding energy $E_{b}$ is defined as:

$$
\begin{gathered}
E_{b}=\mathrm{Au}(\mathrm{III}) / \mathrm{DBA} / \mathrm{Au}(\mathrm{III}) \\
-[E(\mathrm{DBA})+2 E(\mathrm{Au}(\mathrm{III}))] .
\end{gathered}
$$

$E(\mathrm{DBA})$ is the total energy of DBA and $E(\mathrm{Au}(\mathrm{III}))$ and $E(\mathrm{Au}(\mathrm{III}) / \mathrm{DBA} / \mathrm{Au}(\mathrm{III}))$ are total energies of gold cluster and the complex of molecule on the gold clusters, respectively. 
HOMO

LUMO

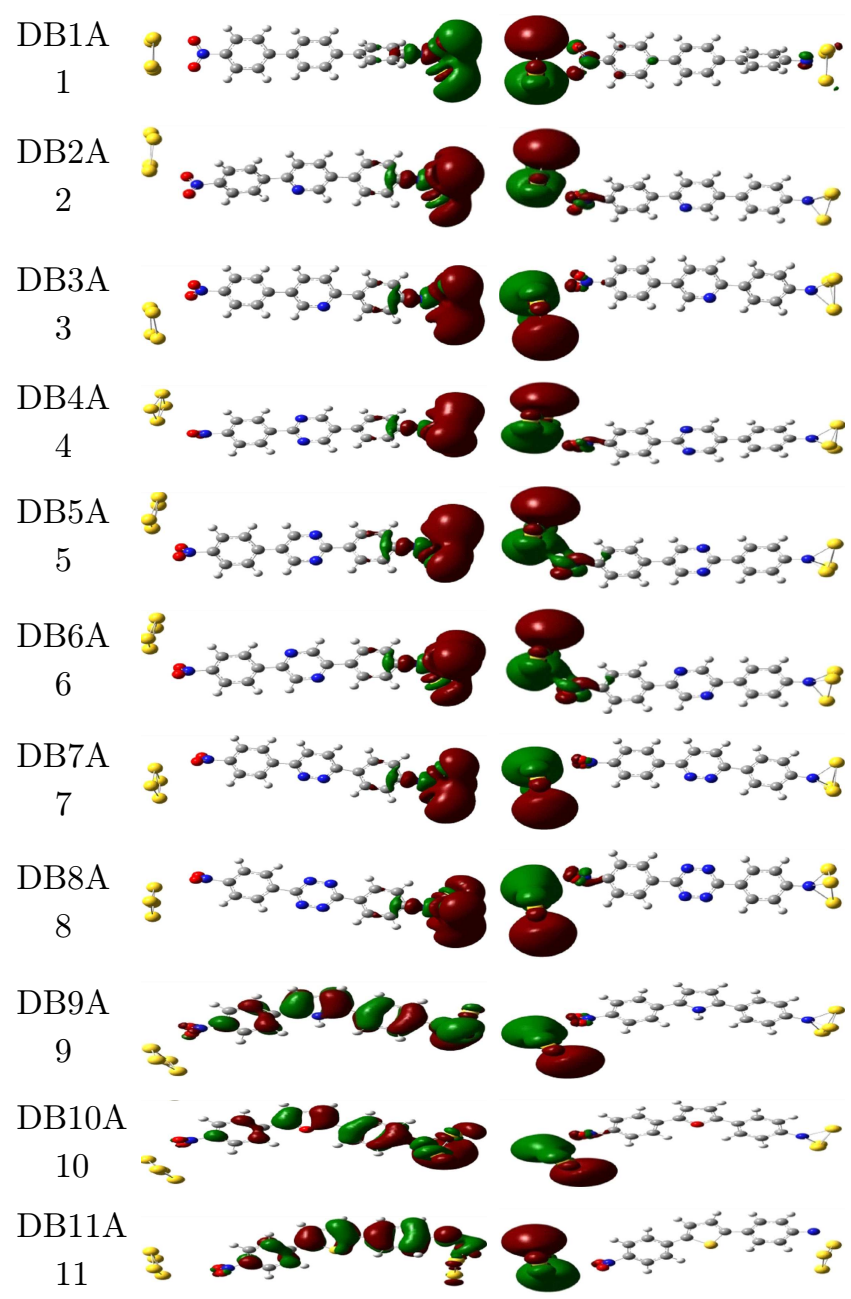

Fig. 3. The shape of HOMO and LUMO orbitals for complexes.

There are several methods to trap gold nanoparticles within the gap. The first reported method is to trap deposited nanoparticles in the gap by applying an alternating electric field [32]. As a representative example, the bond length variation under various EF for complex 1 is shown in Fig. 4. It is known that the bond length alternation (defined as the average difference between the adjacent single and double bonds) along the backbone of

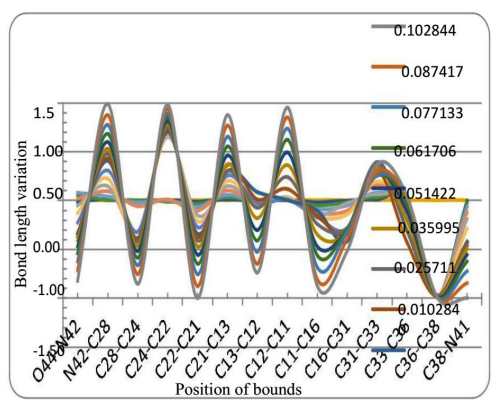

Fig. 4. Bond length evolution for complex 1 under various $E_{F}$ s with respects to the zero $E_{F}$ case. a conjugated system is a crucial parameter for tuning the transportation behavior [33, 34]. At zero field DBAs have longer single bonds and shorter double bond. When $E_{F}$ increases the carbon-carbon single bonds are shortened and the double bonds tend to be elongated, resulting in a decreased bond length alternation. This variation of bond lengths corresponds to the balance of the conjugation within the whole molecule under the interaction with electric field. Moreover, after the application of $E_{F}$, the charges on each atom in the molecule are redistributed. Figure 5 presents the Mulliken charge variation on each atom for complex 1 under the effect of the electric field.

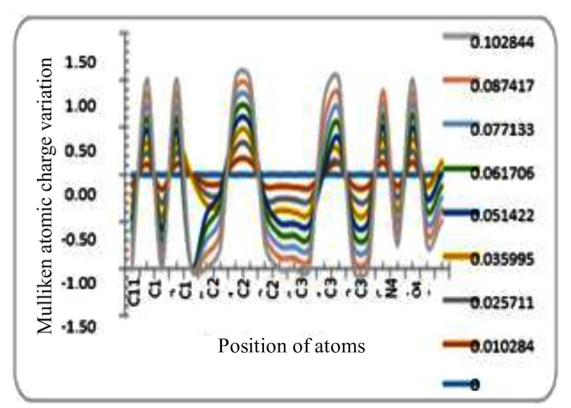

Fig. 5. Mulliken atomic charge variation on each atom for complex 1 under various $E_{F} \mathrm{~s}$ with respect to the zero $E_{F}$ case.

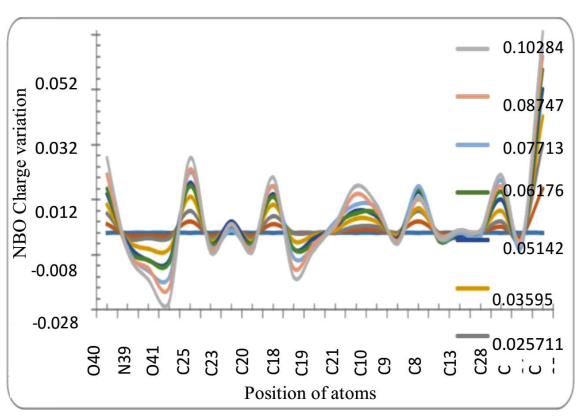

Fig. 6. NBO charge variation on each atom for complex 1 under various $E_{F}$ s with respect to the zero $E_{F}$ case.

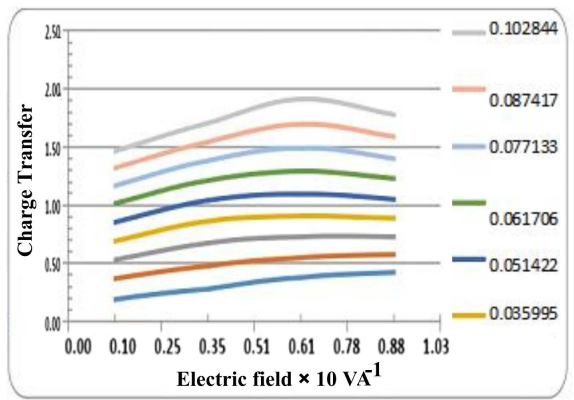

Fig. 7. Electric field effect on the charge transfer for complexes. 
The figure clearly demonstrates the charge transfer from one to the other end of molecule. It is obvious that with the increase in EF the charge on the S1 and S14 increases. Also, we study changes of NBO charge under the various EF on each atom for complex 1 in Fig. 6 . The analyses of charge transfer across the metamolecule interfaces have also been performed at various electric field strengths. Figure 7 shows the variation of charge transfer from the gold cluster to the molecular region versus the $E_{F}$ for complexes 1, 2, and 3. When an electric field is applied, the MOs of molecules are expected to vary. Therefore, it is important to understand how the HOMO, LUMO, and HLG respond to the external field. We can see that HOMO and LUMO shift toward each other with the increase in $E_{F}$ leading to decrease in HLG. The shift of energy levels depends on both the magnitude of the applied field and the interfacial properties. Among three complexes, LUMO and thus HLG of complex 1 show the most pronounced electric field dependence.

\section{Potential barrier for the electron transfer}

From the above discussion, it is clear that though unequal orbital population distribution is observed for different molecules, LUMO (having the extended population all over the molecule) can serve as the conduction channel in the electron transfer process. According to hypothesis, in an electronic circuit Fermi level of the electrode contact lies approximately in the middle of the HLG [35-40].

In a sufficient applied voltage (the applied voltage must be sufficient enough to raise the energy of electron in the Fermi level of contact to that of the LUMO), one electron will be injected from the cathode contact to the LUMO of the molecule. Due to the delocalized nature of the vacant LUMO, the injected electron in the LUMO can travel through the molecule from one end to the other showing the conductance ability of the molecule. This applied voltage, which is required to bring the molecule to behave as a conductor, is called the potential barrier (PB) for conduction. Thus, to gauge the electron transport property in a molecule through frontier molecular orbital analysis, PB for all the molecules was calculated using as shown below [35]:

$$
P B=1 / 2 H L G+q,
$$

where $q=0$ for LUMO as conduction channel. $q=$ $E_{L U M O}$ when the conduction channel is other than LUMO. The quantity $E_{L U M O}$ can be defined as if LUMO is not fully delocalized, the conduction channel will be the still higher unoccupied orbital (LUMO $+n)$, which is fully delocalized all over the molecule and the $E_{L U M O}$ will be equal to $E_{L U M O}+n-E_{L U M O}$. $1 / 2 \mathrm{HLG}$ is the measure of the Fermi level, which according to assumption lies in the middle of HOMO-LUMO gap. As discussed in the previous section, LUMO is the full delocalized low lying vacant orbital for all the molecules, and can serve the channel for conduction process. So using the extreme conditionq $q=0$, for all the molecules, we have calculated the $\mathrm{PB}$ required for the conduction. Along with the HOMO and LUMO orbital energies, PB values for all the molecules are shown in Table IV.

TABLE IV

$\mathrm{PB}$ value $[\mathrm{eV}]$ for molecules.

\begin{tabular}{l|l}
\hline \hline DB1A & 1.560 \\
DB2A & 1.605 \\
DB3A & 1.515 \\
DB4A & 1.645 \\
DB5A & 1.511 \\
DB6A & 1.555 \\
DB7A & 1.572 \\
DB8A & 1.574 \\
DB9A & 1.435 \\
DB10A & 1.442 \\
DB11A & 1.477
\end{tabular}

As it is shown in Table IV, for molecules with electron rich aromatic bridges (molecules 9-11), observed PB was low compared to that of the molecules with electron deficient aromatic bridge (molecules 2-8). Thus in the series, lower $\mathrm{PB}$ values observed were for molecules 9-11 (where the bridging unit was a 5-membered electron rich aromatic ring) and higher PB values for molecules $2-8$, where the bridging unit was a 6 -membered electron rich aromatic ring. The only exception was molecule 5 , which showed a less $\mathrm{PB}$ value. Molecule 5, where the positions of the two $\mathrm{N}$-atoms in the bridge diazine unit nearer to the donor side, created destabilization of HOMO and ultimately reduced the HLG (vice versa for molecule 4, where the $\mathrm{N}$-atoms were nearer to acceptor side). Again, for molecule 1 , due to the maximum twisting at the ring junctions, the $\mathrm{PB}$ value (which was intermediate of the set of molecules 2-8 and 9-11) was obtained. Analysis showed that potential barrier required to achieve the conductions in these aromatic bridged molecules were largely dependent on the differential structural parameters arising due to the presence of the aromatic bridge, but at the same time, the effect of the electron density of the aromatic bridge on the PB also could not be neglected. In other words, the combined effect of structure and the electron density of the bridging aromatic ring were responsible for the stabilization/destabilization of the frontier molecular orbitals and ultimately affected the PB.

\section{Results and discussion}

In the electron transfer process, electronic structure largely controlled the degree of electronic interaction between the D and A by affecting the degree of coupling interactions of the orbitals. In DBA type of systems, where the bridge had the ability to control the communication between the D and A by impacting differential structural orientations, a detailed analysis of the geometric parameters could give a preliminary idea regarding the nature of electronic interaction between the D and A. Various 
aromatic ring bridged DBA types of molecules (molecules 1-11), considered for the study, were shown in Table III. As the ring junctions had the most susceptible geometric parameters, precaution was taken to get the actual minima for all the molecules and the corresponding optimized molecular geometric parameters around the ring junctions obtained from B3LYP/6-31G(d,p) optimized geometries were shown in Table IV. It is clear that during the process of optimization, molecules 8 and 10 turned out to be planar and all other molecules twisted at one or both the ring junctions. Also, it could be seen from the table that the extent of twisting at the bridge junctions varies from bridge to bridge and among all the twisted conformations. The maximum twisting was observed for molecule 1. Maximum twisting for molecule 1 can be attributed to the maximal boundary $\mathrm{CH}-\mathrm{CH}$ interactions near both the ring junctions. For molecules 6 and 7, small twisting was observed at both the ends due to minimal $\mathrm{CH}-\mathrm{CH}$ interactions. For molecule 8, where there was no such $\mathrm{CH}-\mathrm{CH}$ interactions, this turned out to be planar. This was a clear indication of the impact of secondary interactions on deviation of geometry from planarity. During the process of minimization, molecules 1, 4, 5 and 8 turned out to be $\mathrm{C} 2$-symmetric (molecule 8 is $\mathrm{C} 2 \mathrm{~V}$ symmetric) and all other molecules to C1-symmetric. For these molecules which had no symmetry, $-\mathrm{NH}_{2}$ pyramidality was also observed. Analysis of the junction bond lengths showed that, compared to the molecules with 6-membered aromatic bridges (molecules 1-8), reduced in bond lengths were observed for molecules with 5-membered aromatic bridges (molecules 9-11).

This can be explained by considering the electron excess/deficient nature of the central aromatic bridge. It is well known that 5 -membered heterocyclic aromatic compounds are electron rich (6-membered hetero cyclic aromatic compounds are electron deficient) and this favors the easier dissipation of the excess electron density towards the acceptor side resulting in a reduced bond length. It could also be seen that ring junctions bonds for all the molecules showed little double bond characteristics. This tending double bond characteristic indicated the existence of partial coupling of the orbitals at the ring junctions of the molecules (though the two rings in most of the molecules are little out of phase due to twisting).

HOMO-LUMO gap is a key parameter for determining the conductance property. This parameter calculated for molecules and complexes. Natural bond orbital (NBO) (Fig. 7) analysis was carried out in order to explore the nature of $\mathrm{A}-\mathrm{L}$ ( $\mathrm{L}$ was the terminal atom of the linker) interface in greater detail [29]. The orbital character and the occupancy of the bond between $\mathrm{Au}$ and the linker $(\mathrm{A}-\mathrm{L})$ were collected in Table $\mathrm{V}$.

\section{Conclusion}

Through frontier molecular orbital analysis using the DFT formalism, we carried out a systematic study on the DBA molecules with various aromatic bridges placed
TABLE V

Orbital characterization $(s / p / d[\%])$ of $\mathrm{Au}-\mathrm{L}$ bonds.

\begin{tabular}{c|c|c|c|c|c}
\hline \hline Comp. & Occ. & $\mathrm{Au} / \mathrm{L}[\%]$ & $s$ & $p$ & $d$ \\
\hline 1 & 0.772 & $50.95 / 49.05$ & 91.5 & 1.18 & 7.27 \\
2 & 0.79 & $53.71 / 46.29$ & 94.7 & 1.07 & 4.22 \\
3 & 0.78 & $55.19 / 44.81$ & 94.5 & 1.01 & 4.45 \\
4 & 0.80 & $53.98 / 46.02$ & 94.5 & 0.96 & 4.49 \\
5 & 0.79 & $37.90 / 62.10$ & 91.0 & 1.28 & 7.67 \\
6 & 0.81 & $49.55 / 50.45$ & 94.3 & 1.08 & 4.57 \\
7 & 0.80 & $49.77 / 50.23$ & 93.7 & 0.56 & 5.71 \\
8 & 0.95 & $34.85 / 65.15$ & 92.9 & 0.32 & 6.71 \\
10 & 0.76 & $48.38 / 51.62$ & 92.2 & 0.49 & 7.30
\end{tabular}

on the conduction path between the donor and acceptor and through the PB values. we characterized the conduction ability of these molecules. The important observations in this study can be outlined as follows. (1) For all the molecules, the molecular orbital analysis showed that due to the fully delocalized nature of the frontier molecular orbitals (which can be treated as conduction channel) all can be expected to behave as potential molecular wires. (2) When an aromatic bridge was placed in the conduction path between the donor and acceptor, an overall decrease in PB compared to the direct connected donor-acceptor molecule was seen and this suggested the potential usefulness of these bridges. (3) Conductance ability in molecules with electron rich five membered aromatic bridges could be readily achieved at lower PB compared to electron deficient six-membered aromatic bridged molecules. This gives new structureproperty tailoring principle which can be utilized in future design of potential molecular wires. Thus, such structure-property exploitation will be further helpful in designing new polymeric molecular materials for molecular electronics

\section{References}

[1] L.A. Bumm, J.J. Arnold, M.T. Cygan, T.D. Dunbar, T.P. Burgin, L. Jones, D.L. Allara, J.M. Tour, P.S. Weiss, Science 271, 1705 (1996).

[2] M.A. Reed, C. Zhou, C.J. Muller, T.P. Burgin, J.M. Tour, Science 278, 252 (1997).

[3] R.M. Metzger, B. Chen, U. Holpfner, M.V. Lakshmikantham, D. Vuillaume, T. Kawai, X. Wu, H. Tachibana, T.V. Hughes, H. Sakurai, J.W. Baldwin, C. Hosch, M.P. Cava, L. Brehmer, G.J. Ashwell, J. Am. Chem. Soc. 119, 10455 (1997).

[4] D.I. Gittins, D. Bethell, D.J. Schiffrin, R.J. Nichols, Nature 408, 67 (2000).

[5] R.P. Andres, J.O. Bielefeld, J.I. Henderson, B.D. Janes, V.R. Kolagunta, C.P. Kubiak, W.J. Mahoney, R.G. Osifchin, Science 273, 1690 (1997).

[6] V. Capek, T. Mancal, Europhys. Lett. 48, 365 (1999).

[7] G. Ho, J.R. Heath, M. Kondratenko, D.F. Perepichka, K. Arseneault, M. Pezolet, M.R. Bryce, Chem. Eur. J. 11, 2914 (2005). 
[8] N.J. Geddes, J.R. Sambles, D.J. Jarvis, W.G. Parker, Appl. Phys. Lett. 56, 1916 (1990).

[9] A.C. Brady, B. Hodder, A.S. Martin, J.R. Sambles, C.P. Ewels, R. Jones, P.R. Briddon, A.M. Musa, C.A. Panetta, J. Mater. Chem. 9, 2271 (1999).

[10] R.M. Metzger, J.W. Baldwin, W.J. Shumate, I.R. Peterson, P. Mani, G.J. Mankey, T. Morris, G. Szulczewski, S. Bosi, M. Prato, A. Comito, Y. Rubin, J. Phys. Chem. B 107, 1021 (2003).

[11] P. Kornilovich, A.M. Bratkovski, S.-C. Chang, R.S. Williams, US Patent No. 66,70631, 2003.

[12] W.B. Davis, W.A. Svec, M.A. Ratner, M.R. Wasielewski, Nature 396, 60 (1998).

[13] W.B. Davis, M.A. Ratner, M.R. Wasielewski, J. Am. Chem. Soc. 123, 7877 (2001).

[14] S. Sitha, K. Bhanuprakash, J. Mol. Struct. Theochem. 761, 31 (2006).

[15] J.C. Ellenbogen, J.C. Love, Proc. IEEE 88, 386 (2000).

[16] Z. Bayat, S. Daneshnia, S.J. Mahdizadeh, J. Physica E 43, 1569 (2011)

[17] H.Y. Hwang, S.-W. Cheong, N.P. Ong, B. Batlogg, Phys. Rev. Lett. 77, 2041 (1996).

[18] M. Ziese, Phys. Rev. B 60, R738 (1999).

[19] P.K. Siwach, H.K. Singh, O.N. Srivastava, J. Phys. Condens. Matter 20, 273201 (2008)).

[20] J.G. Kushmerick, D.B. Holt, S.K. Pollack, M.A. Ratner, J.C. Yang, T.L. Schull, J. Naciri, M.H. Moore, R. Shashidhar, J. Am. Chem. Soc. 124, 10654 (2002).

[21] K.W. Hipps, Science 294, 536 (2001).

[22] J.M. Beebe, V.B. Engelkes, L.L. Miller, C.D. Frisbie, J. Am. Chem. Soc. 124, 11268 (2002).

[23] Y. Xue, S. Datta, S. Hongy, R. Reifenberger, J.I. Henderson, C.P. Kobiak, Phys. Rev. B 59, R7852 (1999).

[24] S.N. Yaliraki, A.E. Roitberg, C. Gonzalez, V. Mujica, M.A. Ratner, J. Chem. Phys. 111, 6997 (1999).

[25] X. Yin, Y. Li, Y. Zhang, P. Li, J. Zhao, Chem. Phys. Lett. 422, 111 (2006).

[26] K. Prashant, P.K. Jain, J. Phys. Chem. B 110, 723848 (2006).

[27] P.K. Jain, Struct. Chem. 16, 421 (2005).

[28] C. Majumder, T.M. Briere, H. Mizuseki, Y. Kawazoe, J. Chem. Phys. 117, 7669 (2002).
[29] H. Fueno, M. Hayashi, K. Nin, A. Kubo, Y. Misaki, K. Tanaka, Curr. Appl. Phys. 6, 939 (2006).

[30] M.J. Frisch, G.W. Trucks, H.B. Schlegel, G.E. Suzerain, M.A. Robb, J.R. Cheeseman Jr., J.A. Montgomery, T. Vreven, K.N. Kudin, J.C. Burant, J.M. Millam, S.S. Iyengar, J. Tomasi, V. Barone, B. Mennucci, M. Cossi, G. Scalmani, N. Rega G.A. Petersson, H. Nakatsuji, M. Hada, M. Ehara, K. Toyota, R. Fukuda, J. Hasegawa, M. Ishida, T. Nakajima, Y. Honda, O. Kitao, H. Nakai, M. Klene, X. Li, J.E. Knox, H.P. Hratchian, J.B. Cross, V. Bakken, C. Adamo, J. Jaramillo, R. Gomperts, R.E. Stratmann, O. Yazyev, A.J. Austin, R. Cammi, C. Pomelli, J.W. Ochterski, P.Y. Ayala, K. Morokuma, G.A. Voth, P. Salvador, J.J. Dannenberg, V.G. Zakrzewski, S. Dapprich, A.D. Daniels, M.C. Strain, O. Farkas, D.K. Malick, A.D. Rabuck, K. Raghavachari, J.B. Foresman, J.V. Ortiz, Q. Cui, A.G. Baboul, S. Clifford, J. Cioslowski, B. Stefanov, G. Liu, A. Liashenko, P. Piskorz, I. Komaromi, R.L. Martin, D.J. Fox, T. Keith, M.A. Al-Laham, C.Y. Peng, A. Nanayakkara, M. Challacombe, P.M.W. Gill, B. Johnson, W. Chen, M.W. Wong, C. Gonzalez, J.A. Pople, Gaussian 09 (now Gaussian 16), Gaussian Inc., Wallingford (CT) 2016.

[31] S.S. Mallajosyula, J.C. Lin, D.L. Cox, S.K. Pati, R.R.P. Singh, Phys. Rev. Lett. 101, 176805 (2008).

[32] I. Amlani, A.M. Rawlett, L.A. Nagahara, R.K. Tsui, Appl. Phys. Lett. 80, 2761 (2002).

[33] Y. Ye, M. Zhang, J. Zhao, J. Mol. Struct. (Theochem) 822, 12 (2007).

[34] Y. Li, J. Zhao, X. Yin, G. Yin, J. Phys. Chem. A 110, 11130 (2006).

[35] C. Majumder, H. Mizuseki, Y. Kawazoe, J. Phys. Chem. A 105, 9454 (2001).

[36] C. Majumder, T. Briere, H. Mizuseki, Y. Kawazoe, J. Phys. Chem. A 106, 7911 (2002).

[37] H. Mizuseki, K. Niimura, C. Majumder, Y. Kawazoe, Computat. Mater. Sci. 27, 161 (2003).

[38] K. Sohlberg, N. Vedova-Brook, J. Comput. Theo. Nanosci. 1, 256 (2004).

[39] H. Chen, J.Q. Lu, J. Wu, R. Note, H. Mizuseki, Y. Kawazoe, Phys. Rev. B 67, 113408 (2003).

[40] J.C. Ellenbogen, J.C. Love, Proc. IEEE 88, 386 (2000). 